\title{
O perfil sócio-epidemiológico dos hipertensos das Unidades básicas de Saúde
}

Vinicius Lino de Souza Neto. Discente do Curso Bacharelado em Enfermagem da Universidade Federal de Campina Grande e participante do Programa Institucional Voluntário de Iniciação Científica - PIVIC 2012/2013. E-mail: vinolino@hotmail.com.

Polianna Alves Sucupira. Discente do Curso Bacharelado em Enfermagem da Universidade Federal de Campina Grande. E-mail: pollyhankel@hotmail.com

Fernando Silvio de Souza Virgolino. Discente do Curso Bacharelado em Enfermagem da Universidade Federal de Campina Grande. E-mail: fernandovrigolino@ hotmail.com

Ana Elza Oliveira de Mendonça. Enfermeira. Doutoranda em Ciências da Saúde CCS/UFRN. Mestre em Enfermagem pela Universidade Federal do Rio Grande - UFRN. Especialista em Nefrologia pela Universidade Federal de São Paulo - UNIFESP. Professora do curso de Enfermagem da Universidade Federal do Rio Grande do Norte - UFRN. E- mail: a.elza@uol.com.br

José Justino Filho**. Orientador do Programa Institucional Voluntário de Iniciação Cientifica - PIVIC 2012/2013. Mestrado e Doutorado em Sociologia pela Universidade Federal da Paraiba. Professor Associado II do Curso de Enfermagem da Universidade Federal de Campina Grande - UFCG/ Campus- Cuité, Paraíba (PB), Brasil. E-mail: josejustino@bol.com.br

**Endereço para correspondência do autor:

Sitio olho D’água da bica, S\N, CEP: 58.175-000 Tel: 3372-1900. Professor da Graduação em Enfermagem da Universidade Federal de Campina Grande - UFCG/ Campus - Cuité. E-mail: josejustino@bol.com.br

\section{Recebido em: 15/05/2013 - Aprovado em: 30/07/2013 - Disponibilizado em: 15/08/2013}

\section{Resumo}

Objetivo: identificar o perfil sócio epidemiológico dos hipertensos assistidos nas unidades básicas de saúde da zona urbana.Metodologia:estudo exploratório descritivo, e abordagem quantitativa, tendo como população amostral 150 hipertensos, distribuidos entre as cinco unidades básicas de saúde do município do vale do Curimataú, Paraíbano- PB. O projeto foi aprovado conforme parecer do Comitê de Ética em Pesquisa, CAAE No 03454112.5.0000.5182. Resultados e Discussão: a faixa etária dos participantes váriou de 66 a 75 anos 48 (32\%); houve predominância do sexo feminino 107 (71,3\%); casadas 82 (54,7\%); não alfabetizadas 75 (50\%); quanto à raça 85 (56,7\%) eram brancas;residem com filhos (as) e esposo 105(70\%) ;renda salarial estimada em um salário mínimo 98 (65,3\%). O estudo aborda também a questão do vício dos pesquisados, onde 69 (46\%) fazem o uso do tabaco de forma ativa, em relação à prática de atividade física, 120 (80\%) não prática nenhum tipo de atividade, 89 (59,3\%) vão as vezes a unidade de saúde e 60 (40\%) faziam uso da medicação entre 5 á 10 anos. Conclusão:a população estudada, é composta em sua maioria por mulheres, caucasianas, com renda de um salário mínimo, fumantes e sedentárias. Observou-se ainda, que os hipertensos, não comparecem e não costuma buscar os serviços oferecidos na rede primária de atenção a saúde do município estudado.

Descritores: Atenção Básica. Fatores sociais. Hipertensão.Educação em Saúde. Doença Crônica

\section{ABSTRACT}

Objective: To identify the socio demographic characteristics of hypertensive assisted in the basic health zone urbana. Metodologia: exploratory, descriptive, and quantitative approach, taking as sample 150 hypertensive population, 
distributed among the five primary health care units of the municipality Valley Curimataú , Paraiba-PB. The project was approved opinion of the Ethics Committee in Research, CAAE No. 03454112.5.0000.5182.Resultados and Discussion: The age of participants ranged 66-75 years (32\%), there was a predominance of females 107 (71.3\%), married (54.7\%); analfabetizadas not (50\%); regarding race $8656.7 \%$ were white; reside with children (as) and spouse (70\%); wage income estimated at 98 minimum wage $(65.3 \%)$. The study also addresses the issue of addiction of respondents, where $69(46 \%)$ reported tobacco use actively in relation to physical activity, $120(80 \%)$ did not practice any kind of activity, $89(59,3 \%)$ will sometimes the health unit and 60 (40\%) were using medication between 5 will anos.Conclusão 10: The study population is composed mostly of women, Caucasian, earning minimum wage, smoking sedentary. It was also observed that the hypertensive, do not attend and does not usually seek the services offered on the network of primary health care services from the municipality.

Keywords: Primary Care. Social factors. hypertension. Educação in Health Chronic Disease

\section{INTRODUÇÃO}

As doenças do aparelho circulatório constituem as principais causas de morte da população adulta brasileira e de internações hospitalares no âmbito do Sistema Único de Saúde, após parto. Entre as causas de morte, predominam as doenças cerebrovasculares e as doenças isquêmicas do coração entre habitantes com 25 anos de idade (98,2 e 91,5 por 100.000, respectivamente) (BRASIL, 2002). A hipertensão arterial é o principal fator de risco modificável para doença coronariana, doenças cerebrovasculares e insuficiência cardíaca congestiva, entre outras doenças do aparelho circulatório (COSTA; PEIXOTO; FIRMO, 2004).

A hipertensão arterial é considerada um problema de saúde pública por sua magnitude, risco e dificuldades no seu controle. No Brasil, em 2003, 27,4\% dos óbitos foram decorrentes de doenças cardiovasculares, atingindo $37 \%$ quando são excluídos os óbitos por causas mal definidas e a violência (MOLINA et al., 2003). A principal causa de morte em todas as regiões do Brasil é o acidente vascular cerebral, acometendo as mulheres em maior proporção (V DIRETRIZES BRASILEIRA DE HA, 2006).

Na maioria dos casos, desconhece-se a causa da hipertensão arterial, há evidências indicando que a hipertensão arterial essencial do adulto possa ter seu início na infância ou na adolescência. Porém, vários são os fatores que podem estar associados à elevação da pressão arterial como sedentarismo, estresse, tabagismo, envelhecimento, história familiar, raça, gênero, peso e os fatores dietéticos (JARDIM \& COLS,2007).

Acredita-se que as crenças de acordo com as quais as pessoas tendem a viver afetam diretamente os hipertensos na forma como enfrentam a doença e o tratamento dessa enfermidade (PERES; MAGNA; VIANA, 2003).Os Programas de Saúde Pública vêm evidenciando, cada vez mais, a importância da abordagem multiprofissional, uma vez percebida a necessidade dessa forma 
de atuação, levando-se em conta o fato de o cliente, em geral,carecer de intervenções que fogem da competência de um só profissional (MACIEL \& ARAÚJO, 2003).Considerando a necessidade populacional brasileira de forma generalizada o presente estudo teve como objetivo identificar o perfil sócio epidemiológico dos hipertensos assistidos nas unidades básicas de saúde da zona urbana.

\section{MÉTODOS}

A presente pesquisa foi de natureza exploratório-descritivo, com abordagem quantitativa. A pesquisa descritiva observa, registra, analisa e correlaciona fatos ou fenômenos sem manipulá-los, buscando conhecer as diversas situação e relações que ocorrem na vida social, política, econômica e demais aspectos do comportamento humano, tanto dos indivíduos tomado isoladamente como grupos e comunidades nais complexas (CERVO \& BERVIAN, 2002).

O estudo teve como lócus as Unidades Básica de Saúde da zona urbana do município de Cuité, localizado este, na Mesorregião do Agreste Paraibano e na Microrregião do Curimataú ocidental, ficando a $117 \mathrm{~km}$ da cidade de Campina Grande e 235 km da capital João Pessoa, com aproximadamente 24.000 hab, tendo como principal atividade econômica a agropecuária (TEIXEIRA, 2003). O município possui 9 (nove) unidades básicas de saúde da família, estando 5(cinco) localizada na zona urbana e 4 (quatro) em zona rural (BRASIL, 2013). A pesquisa foi desenvolvida entre os meses de Novembro de 2012 á Março de 2013.

Neste contexto, a população da pesquisa foi constituida, pelos hipertensos cadastrados no programa do hiperdia do referido município, que é um programa ou sistema de cadastramento e acompanhamento de pessoas hipertensas e diabéticas que são atendidas nas unidades do Sistema Único de Saúde. O mesmo possibilita mudanças em estratégias de saúde pública para a melhoria da qualidade de vida dessas pessoas e a redução do custo social (BRASIL, 2006).

O quantitativo de hipertensos cadastrados no referido programa chega em um total de aproximadamente 1.360 entre nas unidades básicas de saúde da zona urbana, conforme dados da secretária municipal de saúde do referido município (BRASIL,2013).

A amostra foi delimitada pelos seguintes critérios de inclusão:Ter idade superior a 18 anos; Aceitar livremente participar do estudo; Assinar TCLE (Termo de Consentimento Livre e Esclarecido) ou autorizar impressão dactaloscópica; Está inserido no programa do HIPERDIA e assistido pela equipe multiprofissional das Unidades Básicas de Sáude da zona urbana do município. Como critérios de exclusão da pesquisa optou-se por hipertensos que não aceitaram participar, não fossem portadores 
de surdez, mudez ou problemas psiquiátricos que interferissem na comunicação entrevistado-entrevistador e que fossem maiores de 18 anos, devido a impossibilidades de autorização dos pais.

Dessa forma, depois de aplicados os critérios de inclusão e exclusão a amostra foi composta por 150 hipertensos distribuidos entre as cinco unidades básicas de saúde, salientamos que a amostra corresponde $11,02 \%$ da totalidade da população amostral. $\mathrm{O}$ instrumento de coleta de dados foi um questionário, contendo uma série de 13 perguntas de fundo objetivo. Os dados quantitativos foram analisados estatisticamente tomando por base as variáveis de interesse do estudo, e tabulados ultilizando-se o software Excell, organizados e discutidos, enquadrados de acordo com os fatores sócio-demográfico e epidemiológico (renda, nivel educacional, idade, vício, situação conjugal, convívio familiar entre outros).

A pesquisa foi realizada dentro das normas que regulamentam a pesquisa com seres humanos do Conselho Nacional de Saúde - Ministério da Saúde, de acordo com a resolução No 196 de 10 de outubro de 1996 (BRASIL, 1996).

O estudo foi submetido à plataforma Brasil, conforme legislação vigente, cabendolhe o direcionamento aos Comitês de Ética em pesquisa em seres humanos, para avaliação e licenciamento da efetividade do processo de pesquisa, ao qual a certificação de aprovação ficou a cargo do Comitê de Ética em pesquisa em seres humanos do Hospital Universitário Alcides Carneiro, Campina Grande - PB, tendo aprovação para realização da pesquisa com o parecer numero CAAE: 03454112.5.0000.5182 do referido comitê.

\section{RESULTADOS}

Foram entrevistados $\quad 150$ colaboradores portadores de Hipertensão Arterial inserido dentro do Programa do Hiperdia e assistidos pelo Progama de Saúde da Família na rede de atenção básica do referido município, a presente pesquisa objetiva em levantar as caracteristicas sóciodemografias e epidemiológica da população amostral, tem-se que a idade dos participantes variou de 26 a 95 anos, sendo que $8(5,3 \%)$ estavam na faixa dos 26 a 35 anos, 12 (8\%) entre 36 á 45 anos, 12 (8\%) 46 á 55 anos, 30 (20\%) 56 á 65 anos, 48 (32\%) de 66 á 75 anos, 25 (16,7\%) 76 á 85 anos e pôr fim 15 (10\%) 86 á 95 anos, quanto ao sexo, 107 (71,3\%) dos participantes pertence ao sexo feminino, enquanto $43(28,7 \%)$ são homens. Neste mesmo segmento referente ao estado civil, $82(54,7 \%)$ pessoas são casadas ou viva em união de facto, 12 (8\%) divorciado (a), 40 (26,7\%) víuvo (a) e 16 $(10,6 \%)$ solteria (o),com relação a raça encontrou que $85(56,7 \%)$ caucasiano, 42 
explana a Tabela 1, abaixo:

Tabela 1 - Caracterização dos hipertensos quanto às variáveis sóciodemográficas: idade, sexo, situação conjugal e raça.

$\begin{array}{lcr}\text { 4. Raça } & & \\ \text { Caucasiano (a) } & 85 & 56,7 \\ \text { Negro (a) } & 42 & 28 \\ \text { Pardo (a) } & 23 & 13,3\end{array}$

\begin{tabular}{lrr}
\hline Variáveis & $\mathbf{N}$ & $\%$ \\
\hline 1. Idade (anos) & 8 & 5,3 \\
$26-35$ & 12 & 8 \\
$36-45$ & 12 & 8 \\
$46-55$ & & \\
& 30 & 20 \\
$56-65$ & & 32 \\
$66-75$ & 48 & 10 \\
$76-85$ & & \\
$86-95$ & 25 & 16,7
\end{tabular}

\section{Sexo}

$\begin{array}{lll}\text { Feminino } & 107\end{array}$

$\begin{array}{lll}\text { Msculino } & 43 & 28,7\end{array}$

\section{Situação Conjugal}

Casado (a)/ União de facto $\quad 82 \quad 54,7$

Divorciado (a)

Viúvo (a)

$40 \quad 26,7$

1,3

8,7


foram, $105(70 \%)$ esposo (a) e filhos, 20

$(13,3 \%)$ esposo (a),filhos, mães e netos, 10

$(6,7 \%)$ sozinho (a) e 15 (10\%) esposo(a).

Tabela 2 - Caracterização dos hipertensos quanto às variáveis sóciodemográficas: Nível de Escolaridade, atividade laborativa, renda salarial e com quem vive.

\begin{tabular}{lcc}
\hline Variáveis & N & $\%$ \\
\hline 1. Nível de Escolaridade & & \\
Nível Fundamental I & 40 & 26,7 \\
Nível Fundamental II & 28 & 18,7 \\
Ensino Médio & 7 & 4,6 \\
Não Alfabetizado & 75 & 50
\end{tabular}

2.Ocupação

$\begin{array}{lcc}\text { Empregado (a) } & 15 & 10 \\ \text { Aposentado (a) } & 112 & 74,7 \\ \text { Desempregado (a) } & 23 & 15,3\end{array}$

3. Renda Salarial

$<1$ salário mínimo $\quad 12 \quad 8$

1 salário mínimo $98 \quad 65,3$

2 salários mínimos $\quad 30 \quad 20$

>3 salários mínimos $\quad 10 \quad 6,7$

4. Com quem vive?

Esposo (a) e filhos $\quad 10570$

Esposo (a),filhos, mães e

netos $20 \quad 13,3$

$\begin{array}{lll}\text { Esposo(a) } & 15 \quad 10\end{array}$ $\begin{array}{lll}\text { Sozinho (a) } & 10 & 6,7\end{array}$

Fonte: Dados da Pesquisa, 2013.

Em relação aos dados epidemiológicos a pesquisa indaga quanto ao vício aos hipertensos, pois 69 (46\%) fazem o uso do tabaco de forma ativa, mas 45 (30\%) são exfumante entre 10 a 20 anos de abstinência, 20 $(13,3 \%)$ etilista e $16(10,7 \%)$ não possui nenhum tipo de vício, ainda nessa mesma linha de raciocínio levantou-se o peso dos participantes, onde a média ficou entre 35 a $105 \mathrm{Kg}$, sendo 5 (3,3\%) entre 35 á $45 \mathrm{~kg}, 35$ $(23,3 \%) 46$ á $55 \mathrm{~kg}, 46$ (30,7\%) 56 á $65 \mathrm{~kg}$, 48 (32\%) 66 á 75 kg, 9 (6\%) 76 á 85 kg, 5 (3,4\%) 86 á 95 kg, e 2 (1,3\%) 95 á $105 \mathrm{~kg}$. Os dados denota também quantoao estilo de vida do público alvo, 120 (80\%) não realizam qualquer tipo de atividade física seja regularmente, ou, esporádico, ao quais 30 (20\%) desenvolve algum tipo de atividade. Foi buscado o teor de frequência, ou, procura dos colaboradores a unidade de saúde, ao qual $57(38 \%)$ procura a unidade assiduamente para fazer a revisão sempre que for recomendado, já 89 (59,3\%) vão a unidade ás vezes, ou, nos últimos casos e 4 (2,7\%) nunca ou jamais procura o serviço de saúde, neste mesmo aspecto questionou-se quanto ao tempo de uso do antihipertensivo, onde 35 $(23,3 \%)$ fazem o uso menos que 5 anos, 60 (40\%) entre 5 e 10 anos e $55(36,7 \%)$ á mais de 10 anos toma a medicação, demonstrado na Tabela 3, a seguir: 
Tabela 3 - Caracterização dos hipertensos quanto às variáveis epidemiológicas: vício, peso, estilo de vida, a procura por assitência na unidade e o tempo de uso do Anti - Hipertensivo.

\begin{tabular}{|c|c|c|}
\hline Variáveis & $\mathbf{N}$ & $\%$ \\
\hline \multicolumn{3}{|l|}{ 1. Vício } \\
\hline $\begin{array}{l}\text { Faz uso do tabaco de forma } \\
\text { ativa }\end{array}$ & 69 & 46 \\
\hline $\begin{array}{l}\text { Ex- Fumante, com período de } \\
\text { abstinência entre } 10-20 \text { anos }\end{array}$ & 45 & 30 \\
\hline Etilista & 20 & 13,3 \\
\hline Sem vício & 16 & 10,7 \\
\hline \multicolumn{3}{|l|}{ 2.Peso } \\
\hline $35-45$ & 5 & 3,3 \\
\hline $46-55$ & 35 & 23,3 \\
\hline $56-65$ & 46 & 30,7 \\
\hline $66-75$ & 48 & 32 \\
\hline $76-85$ & 9 & 6 \\
\hline $86-95$ & 5 & 3,4 \\
\hline $96-105$ & 2 & 1,3 \\
\hline
\end{tabular}

3. Realiza algum tipo de atividade física?

Não realiza qualquer tipo de atividade física

12080

Sim, desenvolve algum tipo de atividade

$30 \quad 20$

3. Com que frequência procura a unidade de saúde?

Assiduamente
Ás vezes

8959,3

Nunca ou Jamais

$4 \quad 2,7$

4. A quanto tempo faz o uso do antihipertensivo?

$<$ de 5 anos

$35 \quad 23,3$

5 á 10 anos

$60 \quad 40$

$>10$ anos

$55 \quad 36,7$

Fonte: Dados da Pesquisa, 2013.

Com isso, nesse processo de levantamento de dados diante da população estudada, foi executado a mensuração dos níveis pressóricos, pressão arterial sistólica e diastólica, dos 150 hipertensos, demonstrado de forma sucinta na Tabela 4.

Tabela 4 - Caracterização dos níveis tensionais dos hipertensos assistidos nas Unidades Básicas de Saúde, zona urbana,Cuité, Paraíba, PB, Brasil, 2013.

\begin{tabular}{llll}
\hline $\begin{array}{l}\text { Niveis de Pressão arterial } \\
\text { Sistólica/Diastólica }\end{array}$ & N & $\%$ \\
\hline $\begin{array}{l}\text { Sistólica < } \\
80 \mathrm{mmhg}\end{array}$ & & 28 & 18,7 \\
Sistólica entre 120 á $140 \mathrm{mmhg}$ e diastólica $<$ & & \\
diastólica 80 á $99 \mathrm{mmhg}$ & & & \\
& & 79 & 52,7
\end{tabular}

Sistólica entre 140 á 160 mmhg e diastólica 100 á 110 mmhg $38 \quad 25,3$

Sistólica > 160 mmhg e diastólica > $120 \mathrm{mmhg}$ $5 \quad 3,3$

Fonte: Dados da Pesquisa, 2013. 


\section{DISCUSSÃO}

A prevalência da hipertensão é maior em países desenvolvidos do que em países em desenvolvimento, mas a grande massa populacional em países em desenvolvimento tem contribuído de forma significante para o número total de indivíduos hipertensos no mundo todo. Estima-se que por volta de 2025, 1,5 bilhões de pessoas serão hipertensos

Atualmente, a prevalência média mundial estimada da hipertensão é de 26,4\%, com uma ampla variação dependendo da população estudada, atingindo $21,0 \%$ nos EUA e Canadá, 33,5 a 39,7\% nos países europeus, 15 a21,7\% nos países africanos e asiáticos e cerca de $40 \%$ na América Latina, no Brasil esa incidência variade 24,8 a 44,4\%. No presente estudo, foi realizado o levantamento dos aspectos sóciodemográficos e epidemiológico da população estudada, para assim indentificar o perfil da referida amostra, conforme retrata a Tabela 1 , a faixa etária de maior incidência está entre 66 á 75 anos (32\%), ou seja, pessoas idosas, para, Gus, Fischmann e Medina (2002) os individuos que apresentão-se neste limiar são mais propenso em desenvolver a patologia, devido as alterações fisiológicas na musculatura lisa e no tecido conjuntivo dos vasos, associando o sexo e a raça, os dados retratam que o sexo feminino $(71,3 \%)$ se sobressai diante do sexo oposto e a raça caucasiana $(56,7 \%)$ é preponderante no estudo em questão, para Lessa (2001) a hipertensão arterial na decada de 90 acometia com maior frequência o sexo masculino, porém, devido a mudançados hábitos de vida das mulheres, essa incidência diminuiu progressivamente, decorrente do aumento de mulheres fumantes ativas e o uso de anticoncepcional com níveis elevados de estrogênio, no que tange a raça, o referido autor (a) retrata que a cor negra é a mais vulnerável a hipertensão, com etiologia desconhecida.

Tratando-se da situação conjugal, conforme demonstra o estudo, na Tabela 1, a maioria dos hipertensos são casado e/ou união de facto, tendo como ocupação a aposentadoria, assim retrata a Tabela 2, para alguns estudos, estes relacionam a profissão/ocupação com a elevação da pressão arterial, sendo que os índices mais baixo da pressão arterial ocorrem no grupo socialmente mais privilegiado e os que nunca trabalharam ocupam uma posição intermediária em relação à prevalência de hipertensão (FEIJÃO et al., 2005). A renda dos entrevistados foi mencionada, na Tabela 2, como sendo de 1(um) salário mínimo (65,3\%), uma vez que convivem com cônjuge e filhos (70\%), sendo marido, ou, mulher aposentados e responsáveis pela renda, como convivem com filhos, as despesas geralmente são maiores que as receitas, gerando dificuldades na adesão do tratamento, no que diz respeito as mudanças nos hábitos de vida. 
Quanto à variável do nível de escolaridade (Tabela 2), os dados mostram que a maioria da população amostral são não afalbetizados (50\%), diante disto, Gus, Fischmann e Medina (2002) demonstra que há uma tendência na queda da média da pressão arterial sistólica, conforme o grau de educação aumenta, tudo isso ocorre devido à influência de outros fatores, como aocupação e os fatores de ordem social. Há uma menor prevalência da doença com o aumento do nível de escolaridade, sendo este dado relevante, já que irá interferir diretamente na assimilação das orientações necessárias ao tratamento

Neste mesmo segmento, quanto as váriaveis epidemiológicas, o estudo explana na Tabela 3, de forma sucinta que a maioria dos hipertensos são fumantes ativos (46\%), estão em uma faixa de peso entre 66 á $75 \mathrm{Kg}$ $(32 \%)$ e não realizam qualquer tipo de atividade física $(80 \%)$, com estes fatores de risco, aumenta a sua predisposição para o desenvolvimento da patologia. A prática da atividade física leva a uma homeostásia fisiológica melhorando o funcionamento do organismo, reforçando o coração, músculos, pulmões, ossos e articulação, já a não adesão da prática a atividade, atrelado juntamente ao uso do tabaco leva o individuo ao afloramento de doenças cerebrovasculares e cardiopatias graves (FIELDS et al., 2004).
Dentro do contexto, o estudo em questão, fez o levantamento da frequência do hipertenso a procura de acompanhamento a unidade básica de saúde, neste sentido indentificou que os participantes só vão a unidade as vezes $(59,3 \%)$, conforme vossa necessidade e tomam a mesma medicação á mais de 5 á 10 anos, diante disto e seguindo essa linha lógica de dados, foi mensurado o niveis tensionais dos mesmos, conforme Tabela 4, onde os nivéis pressóricos para sistóle ficou entre 120 á 140 mmhg e os diastólico 80 á 99 mmhg (52,7\%). Com isso, a relação da equipe de saúde com o paciente é fundamental para a adesão do usuário ao tratamento, abordando o mesmo de forma holistica, com a interdisciplinaridade dos profissionais da equipe de moldura multidisciplinar (CHOBANIAN et al., 2003).

Para que os clientes sintam-se motivados a participarem ativamente do tratamento da hipertensão arterial, acredita-se que o caminho seja a educação e a estimulação para a mudança de atitudes diante da patologia. Deve haver também, um maior envolvimento dos profissionais que participam dos programas de atendimento, oferecendo suporte social adequado, através de uma relação social mais próxima, na qual tenha afetividade, comunicação e visão do cliente como único, levando-se em consideração seus problemas e sua história de vida. 


\section{CONCLUSÃO}

Considerando o objetivo do presente estudo de identificar o perfil socioepidemiológico dos hipertensos assistidos nas unidades básicas de sáude do município de Cuité, na Paraíba, podemos desenhar que a população estudada, é composta em sua maioria por mulheres, brancas, idosas, com renda de um salário mínimo, fumantes e sedentárias. Observou-se ainda, que os hipertensos, não comparecem e não costuma buscar os serviços oferecidos na rede primária de atenção a saúde do município estudado.

Por se tratar de uma população idosa, conforme incidências estatísticas, existe uma dificuldade de adesão dessas pessoas ao tratamento, por se tornar mais difícil modificar hábitos de vida de pessoas mais velhas, introduzir alimentação adequada, e fornecer orientações mais coerentes do que as que vem sendo realizadas, por falta de entendimento do indivíduo frente as questões mais fisiológicas, sendo assim afirma-se a necessidade de formação de uma equipe que possa suprir tais faltas, uma vez que os gastos com as incapacidades geradas pelas complicações cardiovasculares são bem maiores para o governo do que criar meios de prevenir, verdadeiramente, com ações válidas e não apenas com a prescrição de medicamentos.

Com isso é nótorio, no referido município, uma deficiência de ações sinérgicas com teor educacional em saúde, frente à população estudada, pois os dados levam a uma ideologia de condicionamento de cartilhas programativas das unidades, embasados na instrumentalização do Ministério da Saúde (MS).

\section{REFERÊNCIAS}

BRASIL. Ministério da Saúde. Secretaria de Informática. Departamento de Informática do Sistema Único de Saúde. Sistema de Informações sobre Mortalidade (SIM), 2000. Brasília (DF); 2002.

BRASIL. Ministério da Saúde. Sistema de Informação da Atenção Básica-Situação de Saúde Paraíba.Disponível: http://tabnet.datasus.gov.br/cgi/tabcgi.exe?siab/cnv/SIABSPB.def $>$ Acesso em 11 de Jan.2013.

BRASIL. Conselho Nacional de Saúde. Resolução nº 196 de 10 de outubro de 1996. Aprova Diretrizes e Normas Regulamentadoras de Pesquisas envolvendo Seres Humanos. 1996b. Disponível em: http://conselho.saude.gov.br/comissao/conep/resolucao.html . Acesso em: 11 jan. 2013. 
BRASIL. Ministério da Saúde. A vigilância, o controle e a prevenção das doenças crônicas não transmissíveis DCNT- no contexto do Sistema Único de Saúde brasileiro - Brasília: Ministério da Saúde; OPAS; 2006.

CERVO, A.L.; BERVIAN, P.A. Metodologia científica. 5 ed. São Paulo: Prentice Hall. p. 242. 2002.

CHOBANIAN, A.V et al. National High Blood Pressure Education Program Coordinating Committee.The Seventh Report of the Joint National Committee on Prevention,Evaluation and Treatment of High Blood Pressure. Hypertension. 42:1206-252. 2003. Disponivel em: http://hyper.ahajournals.org/content/42/6/1206.short. Acesso em : 11 jan.2013.

COSTA, M.F.L.; PEIXOTO, S.V.; FIRMO,J.O.A. Validade da hipertensão arterial auto-referida e seus determinantes (projeto Bambuí). Rev Saúde Publica. 2004; 38(5): 637-42. Disponivel em:http://www.scielo.br/pdf/rsp/v38n5/21750.pdf. Acesso em: 11 jan.2013.

FEIJÃO, A.M.M et al. Prevalência de excesso de peso e hipertensão arterial, em população de baixarenda. Arq Bras Cardiol. 2005; 84(1): 29-33. Disponível em: http://www.scielo.br/pdf/\%0D/abc/v84n1/23002.pdf. Acesso em: 11 jan.2013.

FIELDS, L.E. et al. The Burden of Adult Hypertension in the United States 1999 to 2000. A Rising Tide. Hypertension.2004; 44: 398-404. Disponível em: http://hyper.ahajournals.org/content/44/4/398.short. Acesso em: 11 jan.2013.

V Diretrizes Brasileiras de Hipertensão Arterial.Rev. Bras. Hipertens. 2006;13(4): 256-312. Disponível em: http://www.portalsaudebrasil.com/artigospsb/has001.pdf. Acesso em: 11 jan.2013. 
GUS, I.; FISCHMANN, A.; MEDINA, C. Prevalência dos fatores de risco da doença arterial coronariana no estado do Rio Grande do Sul. Arq Bras Cardiol. 2002.78(5): 484-90. Diponivel em: http://www.scielo.br/pdf/\%0D/abc/v78n5/9377.pdf. Acesso em: 11 jan.2013.

JARDIM E COLS. Hipertensão arterial e fatores de risco. Arq. Bras. Cardiol. 2007; 88(4): 452457.. Disponivel em: http://www.arquivosonline.com.br/2007/8804/pdf/8804015.pdf. Acesso em: 11 jan.2013.

LESSA, I. Epidemiologia da hipertensão arterial sistêmica e da insuficiênciacardíaca no Brasil. Revista Brasileira de Hipertensão. 8: 383-92. 2001. Disponivel em: http://departamentos.cardiol.br/dha/revista/8-4/epidemiologia.pdf Acesso em: 11 jan.2013.

MACIEL, I.C.F.; ARAÚJO, T.L. Consulta de enfermagem: análise das ações junto a programas de hipertensão arterial, em Fortaleza. Rev Latino-am Enfermagem.11(2): 207-14. 2003. http://www.scielo.br/pdf/rlae/v11n2/v11n2a10.pdf. Acesso em: 11 jan.2013.

MOLINA, M.C.B et al. Hipertensão e consumo de sal em população urbana. Rev Saúde Pública 2003;37(6):743-50. Disponivel em: http://www.scielosp.org/pdf/rsp/v37n6/18017.pdf Acesso em: 11 jan.2013.

PÉRES, D.S.; MAGNA, J.M.; VIANA, L.A. Portador de hipertensão arterial: atitudes, crenças, percepções, pensamentos e práticas. Rev Saúde Pública. 2003;37(5):635-42. Disponivel em: http://www.scielo.br/pdf/rsp/v37n5/17480.pdf. Acesso em : 11 jan.2013.

TEIXEIRA, L.M.Informando o Trade Turístico Paraibano: Cuité. Caderno de Turismo, p. 9-11. 2003. 